\title{
NUEVOS REGISTROS DE DISTRIBUCIÓN DE MICRUROIDES EURYXANTHUS AUSTRALIS Y MICRUROIDES EURYXANTHUS NEGLECTUS (SERPENTES: ELAPIDAE), Y SU DISTRIBUCIÓN POTENCIAL EN SINALOA, MÉXICO
}

\author{
NEW DISTRIBUTION RECORDS OF MICRUROIDES EURYXANTHUS AUSTRALIS AND MICRUROIDES EURYXANTHUS \\ NEGLECTUS(SERPENTES: ELAPIDAE), AND THEIR POTENTIAL DISTRIBUTION IN SINALOA, MEXICO
}

\author{
Pedro Uriarte-Garzón ${ }^{1}$, Antonio Esaú Valdenegro-Brito ${ }^{2,4}$, Horacio Bárcenas-RodrígueZ ${ }^{3}$ \& URI \\ OMAR GARCÍA-VÁZQUEZ ${ }^{2 *}$ \\ ${ }^{1}$ Departamento Académico de Ciencias Naturales y Exactas, Universidad Autónoma de Occidente, Unidad Los Mochis, Blvd. Macario Gaxiola y Reyes \\ Heroles $S / N$, Los Mochis, Ahome, Sinaloa. \\ ${ }^{2}$ Laboratorio de Sistemática Molecular, Unidad de Investigación Experimental Zaragoza, Facultad de Estudios Superiores Zaragoza, Universidad \\ Nacional Autónoma de México, Batalla 5 de mayo s/n, Col. Ejército de Oriente, 09230, CDMX, México (Correspondencia: urigarcia@gmail.com). \\ ${ }^{3}$ Facultad de Ciencias, Universidad Nacional Autónoma de México, Circuito interior S/N, Ciudad Universitaria, Coyoacán, Ciudad de México. \\ ${ }^{4}$ Posgrado en Ciencias Biológicas, Centro Tlaxcala Biología de la Conducta. Universidad Autónoma de Tlaxcala, Carretera Tlaxcala-Puebla Km. 1.5, \\ La Loma Xicohténcatl, 90070, Tlax. México. \\ *Correspondencia:urigarcia@gmail.com
}

Abstract.- In an analysis of the distribution of the genus Micruroides from Sinaloa, México, we report new state records that increase the known distribution of this species. Additionally, we evaluate the potential distribution in Sinaloa of the two subspecies that are distributed in the state.

Keywords.- Distribution, Micruroides, new records, Sinaloa.

Resumen.- En un análisis de la distribución del género Micruroides de Sinaloa, México, se reportan nuevos registros estatales que aumentan la distribución conocida de esta especie. Adicionalmente, evaluamos la distribución potencial en Sinaloa, de las dos subespecies que se distribuyen en el estado.

Palabras clave.- Distribución, Micruroides, nuevos registros, Sinaloa.

Micruroides es un género monotípico que se distribuye desde el suroeste de los Estados Unidos en Arizona y Nuevo México, hasta México en Sonora, Sinaloa, Chihuahua, Nayarit y Jalisco (Campbell \& Lamar, 2004; Ahumada-Carrillo et al., 2018). Actualmente, se reconocen tres subespecies: Micruroides euryxanthus euryxanthus, $M$. euryxanthus australis y $M$. euryxanthus neglectus, las dos últimas endémicas de México (Roze, 1974; Slowinski, 1995; Campbell \& Lamar, 2004; Lemos-Espinal \& Smith, 2009: Woolrich-Piña et al., 2016; Cruz-Cuevas et al., 2017).

En Sinaloa, los registros de este taxón han sido escasos. Duellman (1957) lo registra por primera vez a partir de un macho colectado a 26.2 kilómetros al nornoroeste de Mazatlán, que identifica como M. e. australis, considerándolo el registro más sureño de esa subespecie (Zweifel \& Norris, 1955; Duellman,
1957). Roze (1967) reasigna el espécimen colectado por Duellman (1957) a M. e. neglectus, y reporta otro macho a $32.1 \mathrm{~km}$ al norte de Mazatlán, considerándolo el registro más meridional del género Micruroides. Hardy y McDiarmid (1969) y Campbell y Lamar (2004) reconocen a M. e. neglectus como la única subespecie de este taxón con distribución en Sinaloa, y sugieren la posible presencia de M. e. australis en el norte de estado. Meik et al. (2007), reportan una hembra de M. e. neglectus en las cercanías al Municipio de Cosalá, Sinaloa a $100 \mathrm{~km}$ al norte de la localidad tipo (Roze, 1967), representando el segundo registro desde la descripción original, y extendiendo su área de distribución estatal. Más tarde, CruzSáenz et al. (2008), Woolrich-Piña (2016), Cruz-Sáenz et al. (2017) e Ahumada-Carrillo et al. (2018) registran a M. e. neglectus para los estados de Nayarit y Jalisco, extendiendo la distribución conocida para el género. 
Por su parte M. e. australis es mencionada por Boger \& Oliver (1945) como un taxón extendido a lo largo del estado de Sonora, con posible presencia en Sinaloa. Zweifel y Norris (1955) reportan a M. e. australis para el extremo sureste de Sonora, en Guirocoba y Álamos, hasta ese entonces, las localidades más meridionales para esta subespecie (Roze, 1974). Aunque los límites de distribución de las subespecies no están claramente definidos, estas localidades en la porción central de Sonora se encuentran en una zona de intergradación con M. e. euryxanthus (Roze, 1974).

En este estudio, se reportan nuevos registros para Sinaloa del género Micruroides. El espécimen colectado asignado a M. e. australis fue depositado en la colección herpetológica del Museo de Zoología, Facultad de Estudios Superiores Zaragoza, UNAM (MZFZ). Adicionalmente para los ejemplares observados, pero no colectados, se depositó una fotografía en la Colección Digital de Vertebrados de la Facultad de Estudios Superiores Zaragoza, UNAM (MZFZ-IMG). Adicionalmente, con el fin de conocer áreas potenciales de distribución de M. e. australis y M. e. euryxanthus en el estado de Sinaloa, se generaron modelos de distribución para cada subespecie. Se utilizaron todos los registros disponibles de M. e. australis y M. e. euryxanthus en el estado de Sinaloa como puntos de presencia (Tabla 1), la depuración de los registros no eliminó ningún punto ya que no se tienen registros duplicados y la distancia entre los puntos más cercanos fue mayor a $4 \mathrm{~km}$. Se obtuvieron las variables bioclimáticas de WorldClim (Fick \& Hijmans, 2017) a una proyección de $1 \mathrm{~km} 2$ (celdas de 30 segundos) de resolución espacial y se recortaron para delimitar el área de estudio, posteriormente, se realizó un análisis de correlación de Pearson para determinar que variables están fuertemente correlacionadas y descartar aquellas que no proporcionan ninguna información bioclimática ( $>0.7$ coeficiente de Pearson). Utilizamos las variables: Estacionalidad de la temperatura (Coeficiente de variación en \%) BIO 4; Oscilación anual de la temperatura $\left({ }^{\circ} \mathrm{C}\right)$ (Coeficiente entre parámetros 5 y 6) $\mathrm{BIO}$ 7; Temperatura promedio del cuatrimestre más frío BIO 11; Precipitación anual (mm) BIO 12; Precipitación del periodo más seco BIO 14; Precipitación del cuatrimestre más cálido BIO 18 y Precipitación del cuatrimestre más frío BIO 19. Finalmente, se utilizó el algoritmo de máxima entropía empleando el programa MaxEnt (Phillips et al., 2006). Los parámetros utilizados fueron por default, con formato de salida Cloglog, se utilizó el $75 \%$ para calibrar el algoritmo y $25 \%$ para su validación con 500 iteraciones de remuestreo con remplazo (Bootstrap) y regla de umbral de presencia mínima de entrenamiento, y se obtuvieron los modelos de distribución para cada subespecie. Evaluamos cada modelo con la curva ROC (receiver operating characteristics) Parcial (Peterson et al., 2008) en NicheToolbox (Osorio-Olvera et al., 2020), el análisis de realizó con 500 iteraciones, 0.05 de proporción de omisión y $50 \%$ de porcentaje de puntos aleatorios.

El 15 de noviembre de 2011 observamos un individuo de $M$. e. neglectus (MZFZ- IMG-184-85), dentro de los límites del ANP Meseta de Cacaxtla $\left(23.674681^{\circ} \mathrm{N}, 106.583409^{\circ} \mathrm{O}\right.$, altitud 170 ms. n. m), en San Ignacio Sinaloa. Recientemente un segundo ejemplar (MZFZ- IMG-186) de esta subespecie fue observado el 8 de febrero de 2019 dentro de la Reserva Ecológica el Mineral de Nuestra Señora de la Candelaria $\left(24.402900^{\circ} \mathrm{N}, 106.613493^{\circ} \mathrm{O}\right.$, altitud 457 msnm.), Cosalá, Sinaloa. Estas observaciones aumentan la distribución municipal y el límite altitudinal conocido de la subespecie en el centro-sur de Sinaloa.

Table 1. Records of the presence of M. euryxanthus australis and M. euryxanthus neglectus used to perform the potential distribution models.

Tabla 1. Registros de presencia de M. euryxanthus australis y M. euryxanthus neglectus utilizados para realizar los modelos de distribución potencial.

\begin{tabular}{cccl} 
Subespecie & Latitud & Longitud & \multicolumn{1}{c}{ Fuente y localidad } \\
\hline M. e. australis & 25.989748 & -108.419438 & Este estudio (Genaro Estrada, 26 km N de Sinaloa de Leyva) \\
M. e. australis & 26.119785 & -108.65372 & Este estudio (Rancho Machochin, Municipio de El fuerte) \\
M. e. australis & 26.021031 & -109.03768 & Este estudio (Sierra Barobampo) \\
M. e. neglectus & 23.494637 & -106.405947 & Duellman 1957 (26.6 km NE de Mazatlán) \\
M. e. neglectus & 23.542747 & -106.416174 & Roze 1967 (32.1 km N de Mazatlán) \\
M. e. neglectus & 24.365498 & -106.589855 & Meik et al. 2007 (100 km N localidad tipo, municipio Cosalá) \\
M. e. neglectus & 23.674681 & -106.583409 & Este estudio (ANP meseta de Cacaxtla, San Ignacio) \\
M. e. neglectus & 24.4029 & -106.613493 & Este estudio (Reserva Ecológica el Mineral de Nuestra Señora de la Candelaria, municipio de Cosalá) \\
\hline
\end{tabular}


En cuanto M. e. australis, un primer ejemplar (MZFZ- IMG187; Fig. 1) fue encontrado el 19 de agosto de 2016, en una zona de matorral espinoso con elementos de bosque tropical caducifolio en la localidad de Genaro Estrada a $26 \mathrm{~km}$ al noreste de la cabecera municipal de Sinaloa de Leyva $\left(25.989748^{\circ} \mathrm{N}, 108.419438^{\circ} \mathrm{O}\right.$, altitud $118 \mathrm{~m}$ ). El 17 de diciembre de 2016, se registra un segundo individuo (MZFZ-IMG-188; Fig. 1) aproximadamente $28 \mathrm{~km}$ al noroeste de Genaro Estrada. El ejemplar fue observado en una ladera rocosa con vegetación de selva baja caducifolia, dentro del Rancho Machochin $\left(26.119785^{\circ} \mathrm{N}, 108.653720^{\circ} \mathrm{O}\right.$, altitud $248 \mathrm{msnm}$ ), en San Blas, municipio de El Fuerte. Finalmente, un tercer individuo (MZFZ-3740; Fig. 1) fue recolectado el 16 de enero de 2019 en la Sierra de Barobampo $\left(26.021031{ }^{\circ} \mathrm{N}\right.$, 109.03768 ${ }^{\circ} \mathrm{O}$, altitud $196 \mathrm{msnm}$ ). El ejemplar, se encontró entre rocas rodeadas de vegetación de matorral sarco-crasicaule y elementos de selva baja caducifolia. A partir de estos registros se extiende la distribución conocida para esta subespecie en 106 km (línea aérea) al sur de la localidad tipo en Guirocoba, Sonora, México (Fig. 2).

Con base en los registros presentados, M. euryxanthus está presente en seis municipios del estado de Sinaloa; tres de ellos con la presencia de Micruroides e. neglectus y tres en los que se encuentra M. e. australis (Fig. 2). En cuanto a los modelos de distribución potencial, para $M$. e. neglectus el valor arrojado de la ROC parcial fue alto (1.93) por lo que se considera un buen rendimiento del modelo y las variables ambientales con alto porcentaje de contribución son, la oscilación anual de la temperatura $\left({ }^{\circ} \mathrm{C}\right)$ (Coeficiente entre parámetros 5 y 6) (BIO 7) (52.2\%) y la temperatura promedio del cuatrimestre más frío (BIO 11) (32.4\%). Por su parte para M. e. australis el valor arrojado de la ROC parcial fue alto (1.95) y se considera un buen modelo,

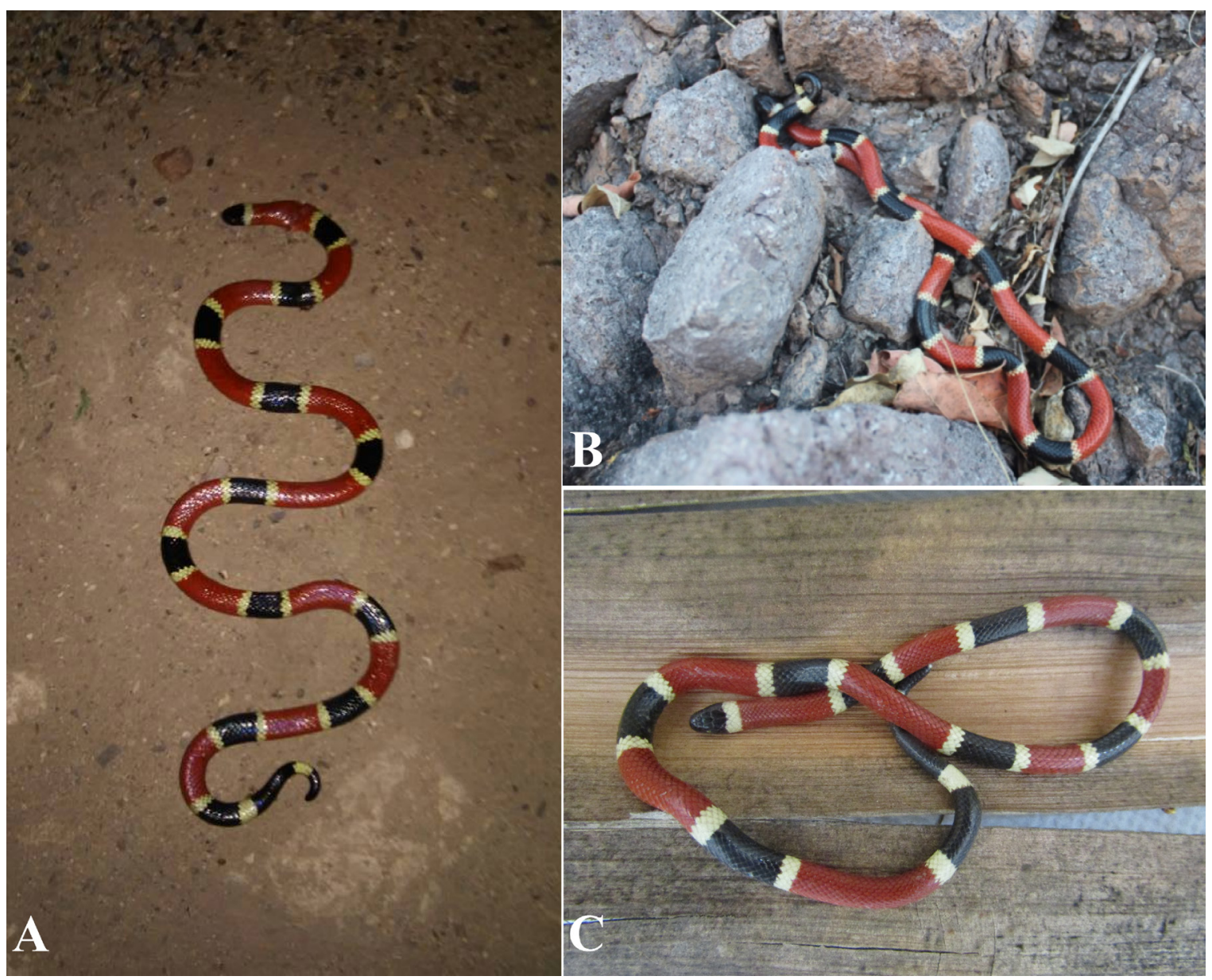

Figure 1. Representatives of Micruroides euryxanthus australis from Sinaloa. A) Genaro Estrada (MZFZ- IMG-187); B) 28 km norteast of Genaro Estrada (MZFZIMG-188); C) Sierra Barobampo, Ahome (MZFZ-3740).

Figura 1. Ejemplares de Micruroides euryxanthus australis en el estado de Sinaloa. A) Genaro Estrada (MZFZ- IMG-187); B) 28 km al noroeste de Genaro Estrada (MZFZ-IMG-188); C) Sierra Barobampo, Ahome (MZFZ-3740). 


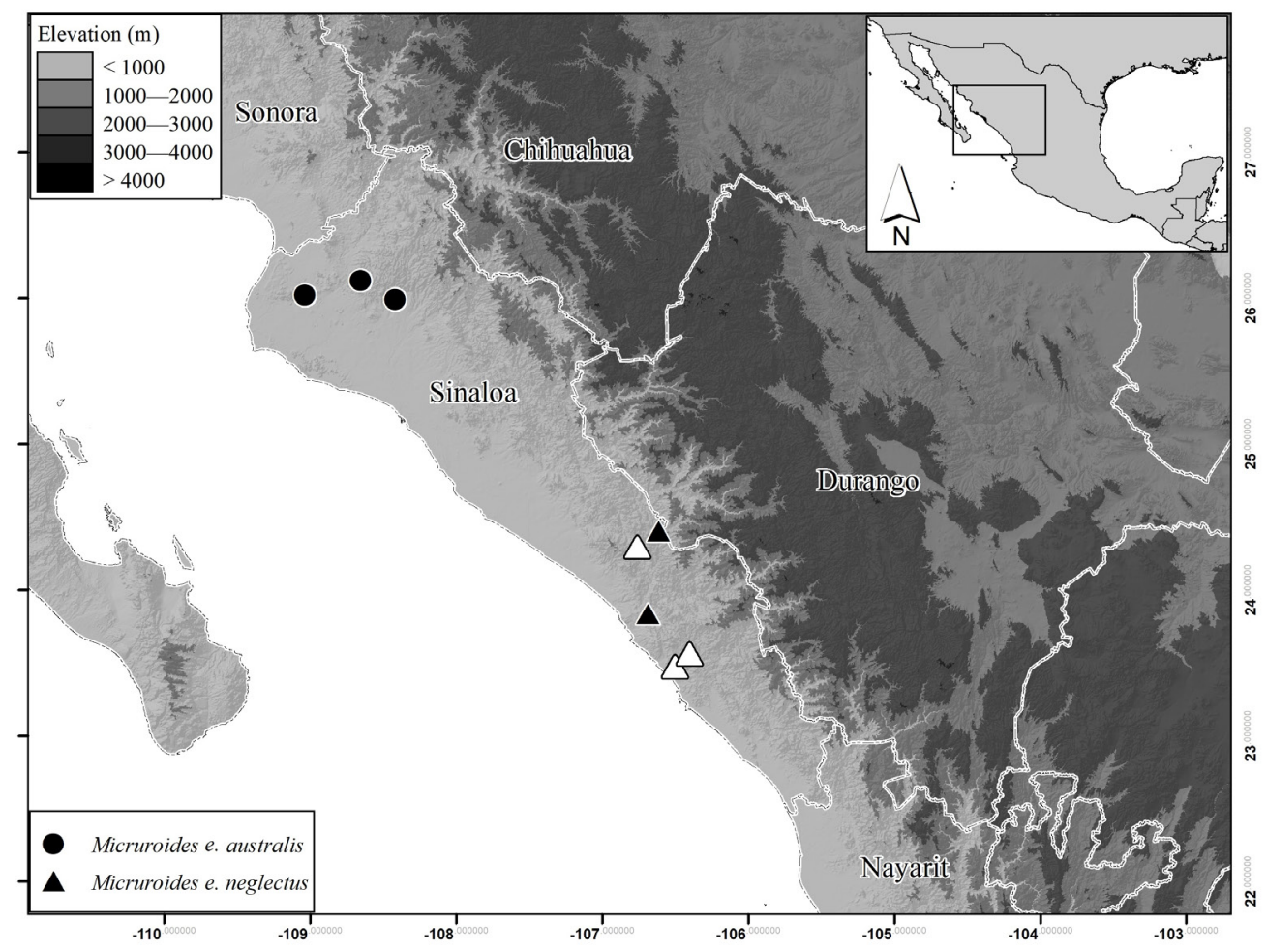

Figure 2. Distribution of Micruroides in Sinaloa state. The black symbols represent the new records and white symbols represent historical records.

Figura 2. Distribución del género Micruroides en el estado de Sinaloa. Los símbolos negros representan los nuevos registros y los símbolos blancos los registros históricos.

las variables ambientales con alto porcentaje de contribución fueron la precipitación del periodo más seco (BIO 14) (68.4\%) y la estacionalidad de la temperatura (Coeficiente de variación en \%) (BIO 4) (12\%). De acuerdo con los índices de idoneidad, la distribución potencial de $M$. e. neglectus parte del centro de Sinaloa hacia el sur para continuar en el estado de Nayarit (Fig. 3). Por su parte la distribución potencial de M. e. australis proviene desde Sonora y va disminuyendo la probabilidad de encontrar a la especie hacía en centro sur del estado de Sinaloa (Fig. 4). En ambos casos la distribución potencial muestra una afinidad biogeográfica, con M. e. australis la Provincia de Sonora y M. e. neglectus a la provincia Pacífica (Morrone et al., 2017). Los registros obtenidos a la fecha y el resultado de los modelos de distribución potencial sugieren que ambos taxones no se encuentran en simpatría en el estado, sin embargo, se requiere trabajo de campo, en particular en el centro del estado para corroborar esta afirmación.

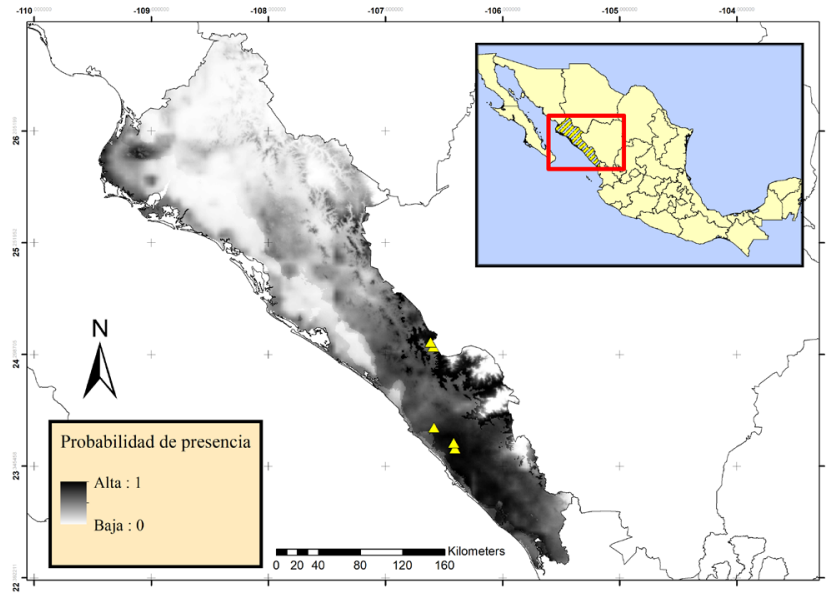

Figure 3. Distribution model of M. e. neglectus in Sinaloa state. The yellow triangles represent ocurres records.

Figura 3. Modelo de distribución de M. e. neglectus en el estado de Sinaloa. Los triángulos amarillos representan los registros de presencia. 


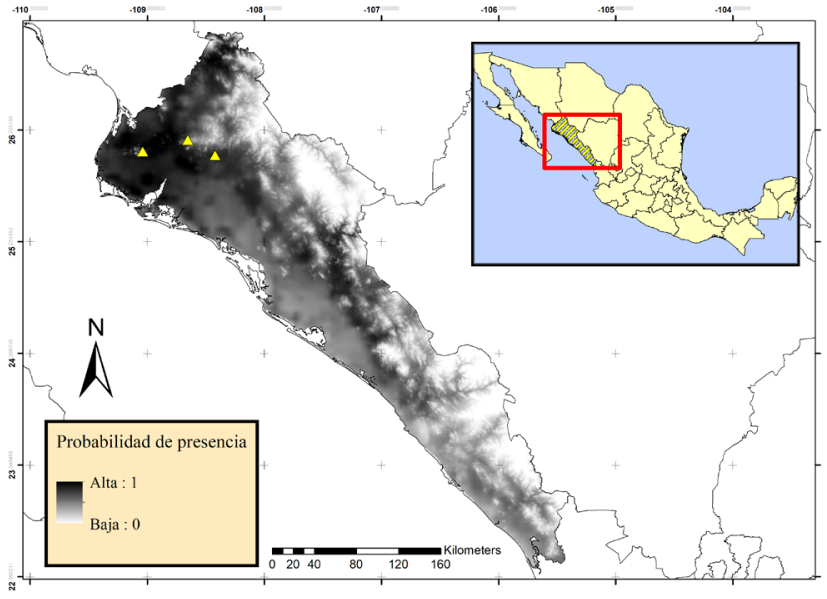

Figure 4. Distribution model of M. e. australis in Sinaloa state. The yellow triangles represent ocurres records.

Figura 4. Modelo de distribución de M. e. australis en el estado de Sinaloa. Los triángulos amarillos representan los registros de presencia.

Agradecimientos.- Parte del trabajo de campo fue financiado por la Dirección General de Apoyo al Personal Académico, Universidad Nacional Autónoma de México (IN-216619) y CONACyT (A1-S-37838) a Uri Omar García-Vázquez. El trabajo de campo fue realizado bajo el permiso de colecta FAUT-0243 otorgado por la Secretaría de Medio Ambiente y Recursos Naturales a Uri Omar García-Vázquez.

\section{LITERATURA CITADA}

Ahumada-Carillo, A., R.A. Carbajal-Márquez, M.A. López-Cuellar \& G.N. Weatherman. 2018. Family Elapidae, The Sonoran Coralsnake, Micruroides euryxanthus (Kennicott, 1860) in the state of Jalisco, Mexico. Mesoamerican Herpetology 5:185-188.

Bezy, R.L., P.C. Rosen, T.R. Van Devender \& E.F. Enderson. 2017. Southern distributional limits of the Sonoran Desert along the mainland coast of northwestern Mexico. Mesoamerican Herpetology 4:138-167.

Boger, C.M. \& J.A. Oliver. 1945. A preliminary analysis of the herpetofauna of Sonora. Bulletin of the American Museum of Natural History 83:297-426.

Campbell, J.A. \& W.W. Lamar. 2004. The venomous reptiles of the western hemisphere. Cornell Univ. Press, Ithaca, New York, USA.

Cruz-Sáenz, D., C.E. Gudiño-Larios, C.D. Jimeno-Sevilla, R.L. Velázquez, \& J. Cortés-Aguilar. 2008. Guía de Reptiles y Anfibios de Arcediano. Comisión Estatal de Agua, Gobierno de Jalisco, Guadalajara, Jalisco, México.

Cruz-Sáenz, D., F.J. Muñoz-Nolasco, V. Mata-Silva, J.D. Johnson, E. García-Padilla, \& L.D. Wilson. 2017. The herpetofauna of Jalisco, Mexico: composition, distribution, and conservation status. Mesoamerican Herpetology 4:22-118.

Duellman, W.E. 1957. Notes of snakes from the mexican state of Sinaloa. Herpetologica 13:237-240.

Fick, S.E. \& R.J. Hijmans. 2017. WorldClim 2: new 1-km spatial resolution climate surfaces for global land areas. International Journal of Climatology 37: 4302-4315

Hardy, L.M. \& R.W. McDiarmid. 1969. The amphibians and reptiles of Sinaloa, México. University of Kansas Publications of the Museum of Natural History 18: 39-252.

Lemos-Espinal, J.A. \& H.M. Smith. 2009. Claves para los anfibios y reptiles de Sonora, Chihuahua y Coahuila, México. Universidad Nacional Autónoma de México-University of Colorado at Boulder-Comisión Nacional para el Conocimiento de la Biodiversidad, México.

Meik, J.M., E.N. Smith \& A.A. Mendoza. 2007. Rediscovery of the rare coralsnake Micruroides euryxanthus neglectus (Serpentes: Elapidae). Herpetological Review 38:293-294

Morrone, J.J., T. Escalante \& G. Rodríguez-Tapia. 2017. Mexican biogeographic provinces: Map and shapefiles. Zootaxa 4277:277279.

Osorio-Olvera, L., A. Lira-Noriega, J. Soberón, A. TownsendPeterson, M. Falconi, R.G. Contreras-Díaz, E. Martínez-Meyer, V. Barve \& N. Barve. 2020. Ntbox: an R package with graphical user interface for modeling and evaluating multidimensional ecological niches. Methods in Ecology and Evolution 11: 11991206.

Peterson, A.T., M. Papeç \& J. Soberón. 2008. Rethinking receiver operating characteristics analysis applications in ecological niche modeling. Ecological modelling 213:63-72

Phillips, S.J., R.P. Anderson \& R.E. Schapire. 2006. Maximum entropy modelling of species geographic distributions. Ecology Modelling 190:231-259 
Roze, J.A. 1967. A check list of the new world venomous coral snakes (Elapidae) with descriptions of new forms. American Museum Novitates 287:1-60

Roze, J.A. 1974. Micruroides, M. euryxanthus. Catalogue of American Amphibians and Reptiles 163:1-4.

Slowinski, J.B. 1995. A phylogenetic analysis of the new world coral snakes (Elapidae: Leptomicrurus, Micruroides and Micrurus) based on allozymin and morphological characters. Journal of Herpetology 29:325-338.

Woolrich-Piña, G.A., P. Ponce-Campos, J. Loc-Barragán, J.P. Ramírez-Silva, V. Mata-Silva, J.D. Johnson, E. GarcíaPadilla \& L.D. Wilson. 2016. The herpetofauna of Nayarit,
Mexico: composition, distribution, and conservation status. Mesoamerican Herpetology 3:375-448.

Webb, R.G., 1984. Herpetogeography in the Mazatlán-Durango region of the Sierra Madre Occidental, Mexico. Pp. 217-241. En Seigel, R.A., L.E Hunt, J.L. Knight, L. Malaret \& N.L. Zuschlog (Eds.), Vertebrate Ecology and Systematics. A Tribute to Henry S. Fitch. University of Kansas Museum of Natural History Special Publication 10.

Zweifel, R.G \& K.S. Norris. 1955. Contribution to the herpetology of Sonora, México: descriptions of new subspecies of snakes (Micruroides euryxanthus and Lampropeltis getulus) and miscellaneous collecting notes. The American Midland Naturalist 54:230-249. 